\title{
Intraepidermal epidermotropic metastatic melanoma: a clinical and histopathological mimicker of melanoma in situ occurring in multiplicity
}

\begin{abstract}
The distinction between primary melanoma and melanoma metastatic to the skin has major prognostic implications. We report a case of a 67 -year-old male with a diagnosis of a superficial spreading melanoma (stage IB) rendered 6 years earlier who presented clinically with an atypical nevus on his left thigh. Histopathological examination showed an intraepidermal melanocytic proliferation that was interpreted as melanoma in situ. Subsequently, 45 additional pigmented macules appeared in crops over a 9-month period. Clinically and dermoscopically, these lesions were extremely polymorphic.

Histopathological findings were compatible with melanoma in situ, as each lesion consisted of a wholly intraepidermal proliferation of markedly atypical melanocytes arranged singly and in nests. A complete gastrointestinal study showed multiple pigmented metastatic lesions throughout the stomach and small bowel, which supported a diagnosis of metastatic melanoma with gastrointestinal and epidermotropic skin involvement. Monosomy of chromosome 9 and a BRAF V600E mutation were detected in the primary tumor sample and in macro-dissected secondary lesions. No CDKN2A or CDK4 germline mutations were found. Intraepidermal epidermotropic metastases of melanoma have been rarely described in literature. In this case, histopathology alone was insufficient to distinguish metastatic melanoma from multiple in situ melanomas. The recognition of epidermotropic metastases should be based on the correlation between clinical, dermoscopic, histopathological and molecular findings.
\end{abstract}

Keywords: atypical features, cutaneous metastases, dermoscopy, epidermotropism, malignant melanoma

Lestre S, João A, Ponte P, Peixoto A, Vieira J, Teixeira MR, Fidalgo A. Intraepidermal epidermotropic metastatic melanoma: a clinical and histopathological mimicker of melanoma in situ occurring in multiplicity.

J Gutan Pathol 2011; 38: 514-520. (C) 2011 John Wiley \& Sons A/S.
Sara Lestre ${ }^{1}$, Alexandre João ${ }^{1}$, Pedro Ponte ${ }^{1}$, Ana Peixoto², Joana Vieira ${ }^{2}$, Manuel R. Teixeira $^{2,3}$ and Ana Fidalgo 1

${ }^{1}$ Dermatology Department, Hospital Santo António dos Capuchos, Centro Hospitalar de Lisboa-Central, Lisbon, Portugal

${ }^{2}$ Genetics Department, Portuguese Oncology Institute, Porto, Portugal, and

${ }^{3}$ Biomedical Sciences Institute (ICBAS),

Porto University, Porto, Portugal

Sara Lestre,

Dermatology Department, Hospital Santo António dos Capuchos,

Alameda Santo António dos Capuchos,

1169-050 Lisbon, Portugal

Tel: +351213136300

Fax: +35121 3562208

e-mail: saralestre@gmail.com

Accepted for publication December 20, 2010 


\section{Intraepidermal epidermotropic metastatic melanoma}

Cutaneous melanoma represents a lethal skin cancer that is responsible for $80 \%$ of skin cancer mortality. ${ }^{1}$ Metastatic melanoma progression typically evolves from local cutaneous growth to regional metastasis involving lymph node(s) or in-transit metastasis, and later disseminated metastatic disease may involve skin or any visceral organ. ${ }^{2}$ Cutaneous melanoma metastases usually involve the dermis and subcutaneous tissue and spare the overlying epidermis. ${ }^{3}$ Rarely, metastatic melanomas can show prominent epidermotropism, mimicking primary melanoma. Epidermotropic metastatic melanoma represents a recognized pathological variant. Its recognition is particularly challenging as its clinical and histopathological appearance can simulate primary melanoma. ${ }^{4}$ This distinction is crucial in the prognosis and treatment approach to affected patients. In 1978, Kornberg and colleagues proposed histopathological criteria for the diagnosis of epidermotropic metastatic melanoma, ${ }^{5}$ which are still used at the present time. However, in the past few decades, the morphological spectrum of epidermotropic metastatic melanoma has broadened and additional criteria have been added. ${ }^{4,6-8}$

Herein, we report a patient whose melanoma recurred with 46 epidermotropic metastases that were wholly intraepidermal. The patient also suffered from multifocal asymptomatic gastrointestinal metastases.

\section{Case report}

In 2002, a 67-year-old male was diagnosed with a non-ulcerated superficial spreading melanoma (stage IB, T2 N0 M0, Breslow's thickness 1.3 mm, Clark's level III) involving the lower back (Fig. 1). Sentinel lymph node evaluation was not performed. After wide excision $(1 \mathrm{~cm})$, adjuvant immunotherapy with

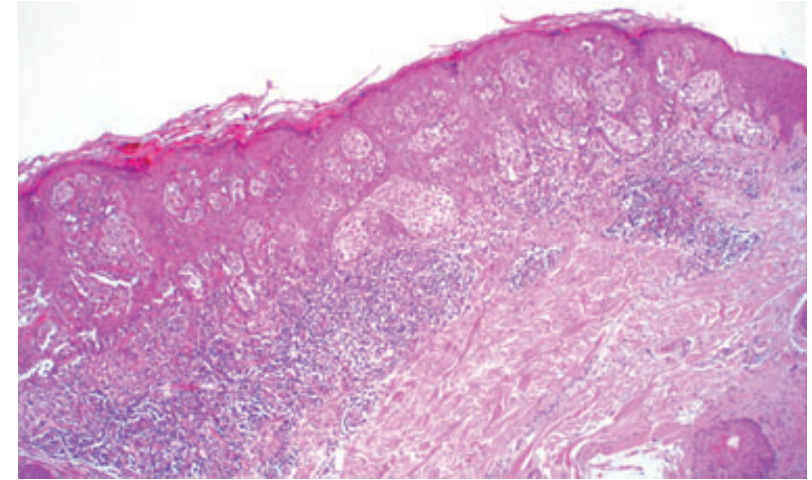

Fig. 2. Histopathology of a pigmented lesion of the thigh that appeared 6 years after the excision of the primary melanoma. There was a wholly intraepidermal proliferation of atypical melanocytes with pagetoid spread, indistinguishable from melanoma in situ (hematoxylin and eosin, $\times 40$ ).

interferon-alpha (3 millions IU, thrice weekly) was started and maintained over 12 months. He was regularly followed and remained free from disease recurrence up until February 2009, when an irregularly shaped and highly pigmented macule appeared on his left thigh. His environmental exposure history was significant for several sunburns before 20 years of age and family history was remarkable for melanoma in two siblings.

Histopathological examination of the new pigmented lesion was interpreted as melanoma in situ (Fig. 2). Subsequently, several small pigmented macules appeared in crops on the back (Fig. 3). The most irregular ones were excised and all biopsy specimens showed markedly atypical melanocytes arranged in irregular nests and solitary units at all levels of epidermis, mimicking melanoma in situ. Neither dermal involvement nor invasion of vascular or lymphatic spaces was detected (Fig. 4). Those judgments were supported by Melan-A and CD31 stains.
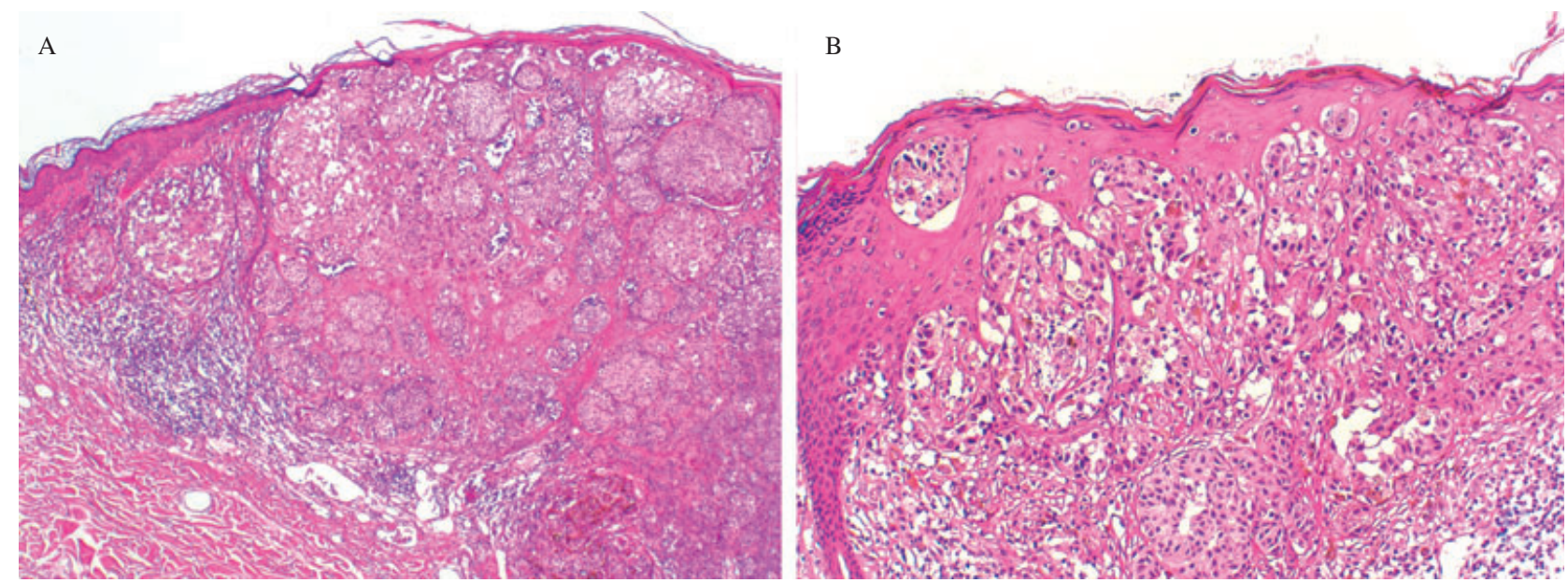

Fig. 1. Histopathology of the primary melanoma of the back, which was excised in November 2002. The proliferation was broad and markedly asymmetrical (A: hematoxylin and eosin, $\times 40$ ). There was a population of atypical melanocytes with pagetoid spread and epidermal consumption $(\mathrm{B}$ : hematoxylin and eosin, $\times 100)$. 


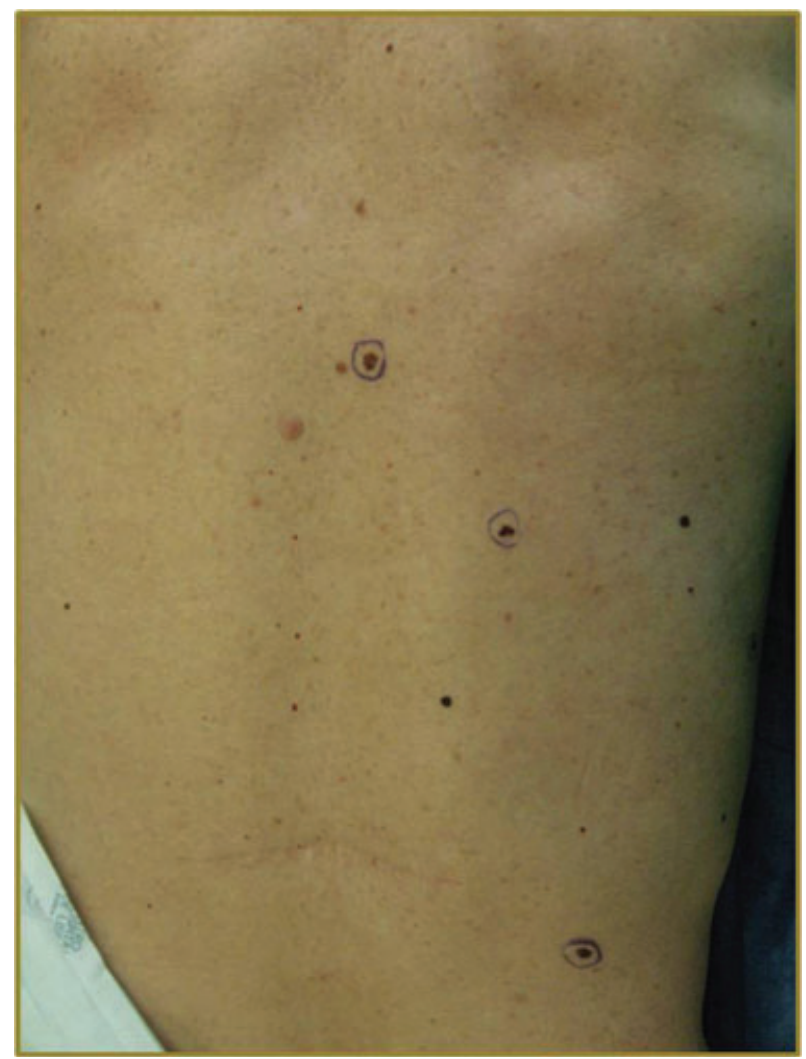

Fig. 3. The clinical appearance of several pigmented lesions on the back is depicted.

New crops of pigmented lesions appeared on the back, arms and legs. Most of them were small (less than $5 \mathrm{~mm}$ of diameter), brownish, well-defined, symmetric and macular. A total of 46 lesions were excised during a 9-month period. Histopathological findings were similar in all specimens, consisting of an exclusively intraepidermal proliferation of large atypical melanocytes with increased nuclear to cytoplasmatic ratio and prominent nucleoli. Those findings were compatible with melanoma in situ, although many lesions were small, symmetric and circumscribed (Fig. 5).

Dermoscopically, the lesions were extremely polymorphic. Most of the excised macules had a lightbrown symmetric homogeneous pattern with only few darker dots at the periphery. Frequently, a regular starburst pattern was also observed. In some lesions, melanoma-specific criteria were present, particularly blue-white structures and irregular blotches (Fig. 6).

The patient underwent staging with inguinal nodal ultrasonography; computed tomography $(\mathrm{CT})$ of the head, chest, abdomen and pelvis; brain magnetic resonance (MR) and positron emission tomography/CT (PET-CT). Distant metastases were not identified by this testing. Results of routine laboratorial evaluation, including lactate dehydrogenase $(\mathrm{LDH})$ and $\mathrm{S}-100$ protein, were unremarkable. Mucosal and uveal involvement by melanoma was also excluded. Even though the patient was asymptomatic, an upper endoscopy was performed that showed pigmented macules and ulcerated nodules involving the stomach and duodenum mucosa. The biopsy specimens revealed metastatic melanoma within superficial mucosa (Fig. 7). This diagnosis was supported by positive immunostaining with HMB-45. Surgical resection of the stomach and proximal duodenum was considered, but surgery was rejected after detection of multifocal metastatic melanoma throughout the entire length of the jejunum and ileum by capsule videoendoscopy.

Molecular analysis was also performed. Genomic DNA was extracted from peripheral blood nucleated cells using conventional procedures. No germline point mutations or exonic rearrangements were found in the $C D K N 2 A$ gene or point mutations in CDK4 (codon 24). Fluorescence in situ hybridization (FISH) analysis was completed using paraffinembedded sections of the primary lesion and a commercially available probe to target the gene $C D K N 2 A$ in chromosome band 9p21 and the centromere of chromosome 9 (LSI CDKN2A /CEP 9 Probe Vysis/Abbott). Monosomy of chromosome 9 (including loss of a copy of the $C D K N 2 A$ gene) was found in the primary tumor. We also performed mutation analysis of BRAF (codon 600) in paraffin-embedded sections of the primary tumor sample and in freshly collected biopsy specimens from seven presumed secondary lesions of the skin. A BRAF V600E mutation was detected in the primary tumor sample but not in the seven skin biopsy specimens analyzed. Given this unexpected finding, we looked for monosomy 9 and $B R A F$ codon 600 in paraffin-embedded tissue sections of five distinct and separate secondary skin lesions (molecular analysis was completed after macrodissection to avoid contamination with normal cells) and indeed found that the primary tumor and all the analyzed secondary lesions shared the chromosome 9 monosomy and BRAF V600E mutation.

During ongoing follow up, albeit with the continuous appearance of new pigmented lesions until November 2009, the patient remained asymptomatic with good general status. A multidisciplinary team decided to delay initiation of systemic chemotherapy and/or chemoimmunotherapy and wait for the beginning of a new clinical trial recruiting patients with stage IV malignant melanoma.

In February of 2010, the patient died as a result of a large aortic thrombus with an extensive systemic embolization affecting the brain, heart, spleen and kidney. The patient's family refused an autopsy. 


\section{Intraepidermal epidermotropic metastatic melanoma}
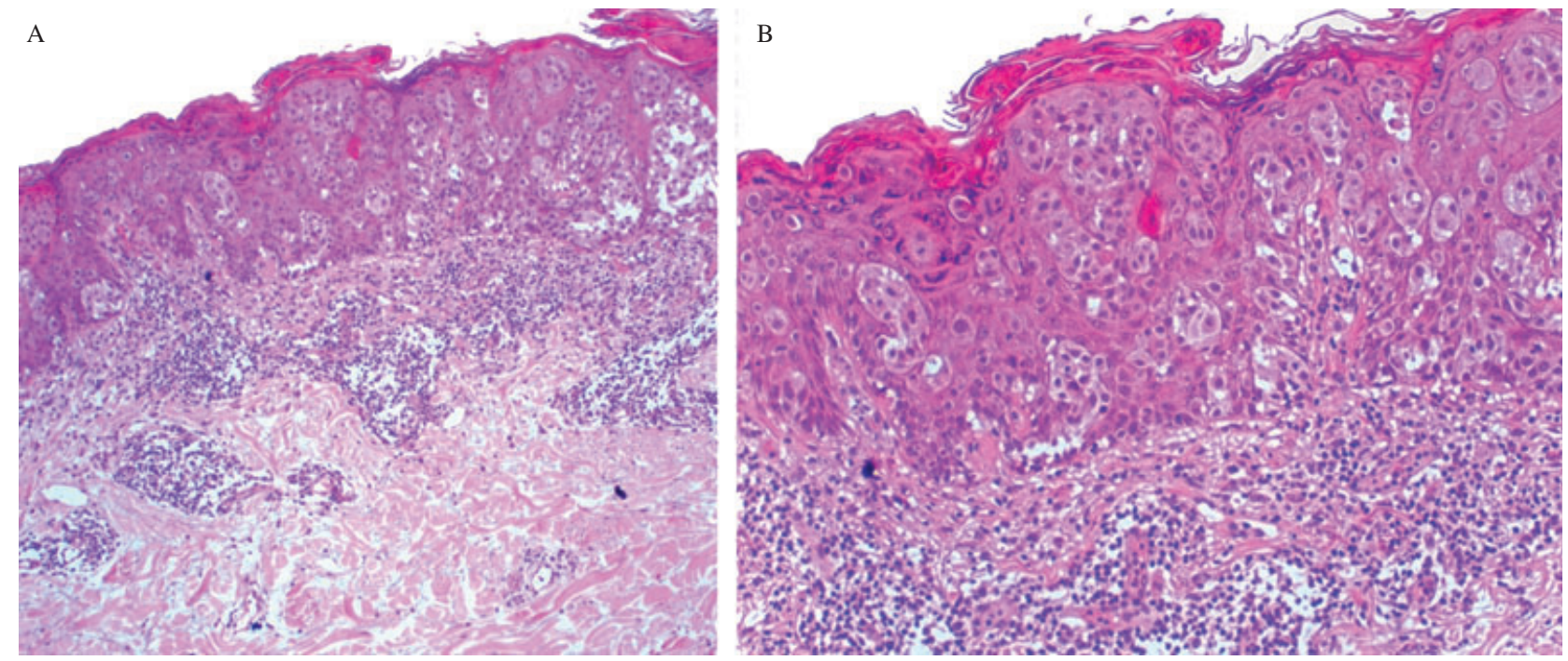

Fig. 4. The histopathology of a pigmented macular lesion from the lower back (April 2009) is illustrated. There is an atypical intraepidermal melanocytic proliferation with an associated lymphocytic infiltrate in the superficial dermis (A: hematoxylin and eosin, $\times 40$ ). Further detail shows atypical melanocytes arranged in singly and in nests (B: hematoxylin and eosin, $\times 100)$.

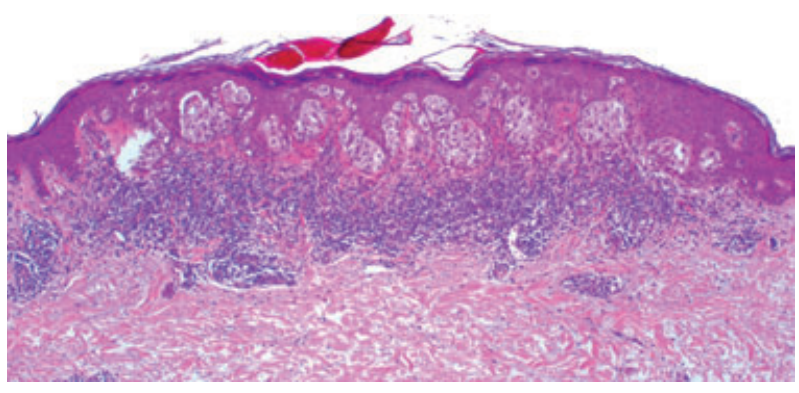

Fig. 5. The histopathological features of a pigmented skin removed from the right thigh (July 2009). There is a small, symmetric and well-circumscribed lesion composed of nests of atypical melanocytes confined to the epidermis.

\section{Discussion}

Cutaneous metastases of melanoma have a variable appearance. Papules, nodules and tumors commonly involve the dermis and subcutis and less frequently involve the overlying epidermis. ${ }^{3}$ Metastases with epidermal involvement have been designated epidermotropic metastatic melanoma and usually present as multiple, symmetric, small lesions occurring in crops located in the same region as the primary melanoma. Less frequently, widely disseminated lesions can be seen. ${ }^{3-6}$

Histopathological criteria for defining epidermotropic metastatic melanoma have evolved over the years. The distinction between metastatic and primary melanoma was initially based on the absence of involvement of the overlying epithelium. ${ }^{9}$ In 1978, Kornberg and colleagues developed specific histopathological features that favored the diagnosis of epidermotropic metastatic melanoma, including: (a) dermal involvement equal or greater than epidermal involvement; (b) junctional epidermal involvement not extending beyond the edge of the dermal component; (c) atypical melanocytes filling the papillary dermis with thinning of epidermis; (d) widening of papillae with the formation of an epithelial collarette; and (e) the identification of atypical melanocytes in vascular and lymphatic spaces. ${ }^{5}$ Subsequently, epidermotropic metastatic melanoma with pagetoid spread of atypical melanocytes beyond the lateral limits of the dermal component of dermal melanoma was described. ${ }^{6,10,11}$ Several reports also suggested that angiotropism is suggestive of metastatic disease. ${ }^{7,12}$

Immunohistochemistry may be useful as a tool for distinguishing epidermotropic metastatic melanoma and multiple primary melanomas in situ, but the approach lacks specificity: metallothionein expression is strongly expressed by epidermotropic metastatic melanoma but is also expressed by Spitz nevi. In epidermotropic metastases, the intensity of HMB-45 staining is greater than that of S100 , but this phenotype does not exclude primary melanoma. ${ }^{4,13}$

Abernethy et al. reported two patients with widespread epidermotropic metastatic melanoma, including classic lesions with intraepidermal extension beyond the intradermal component and 10 lesions with only epidermal involvement simulating melanoma in situ. ${ }^{13}$ In contrast to previous reports, our patient's lesions all showed identical histopathology with exclusively epidermal involvement and no detectable dermal disease. In this setting, the distinction between epidermotropic metastatic melanoma and multiple primary melanomas is virtually impossible by light microscopy. However, the diagnosis of 


\section{Lestre et al.}
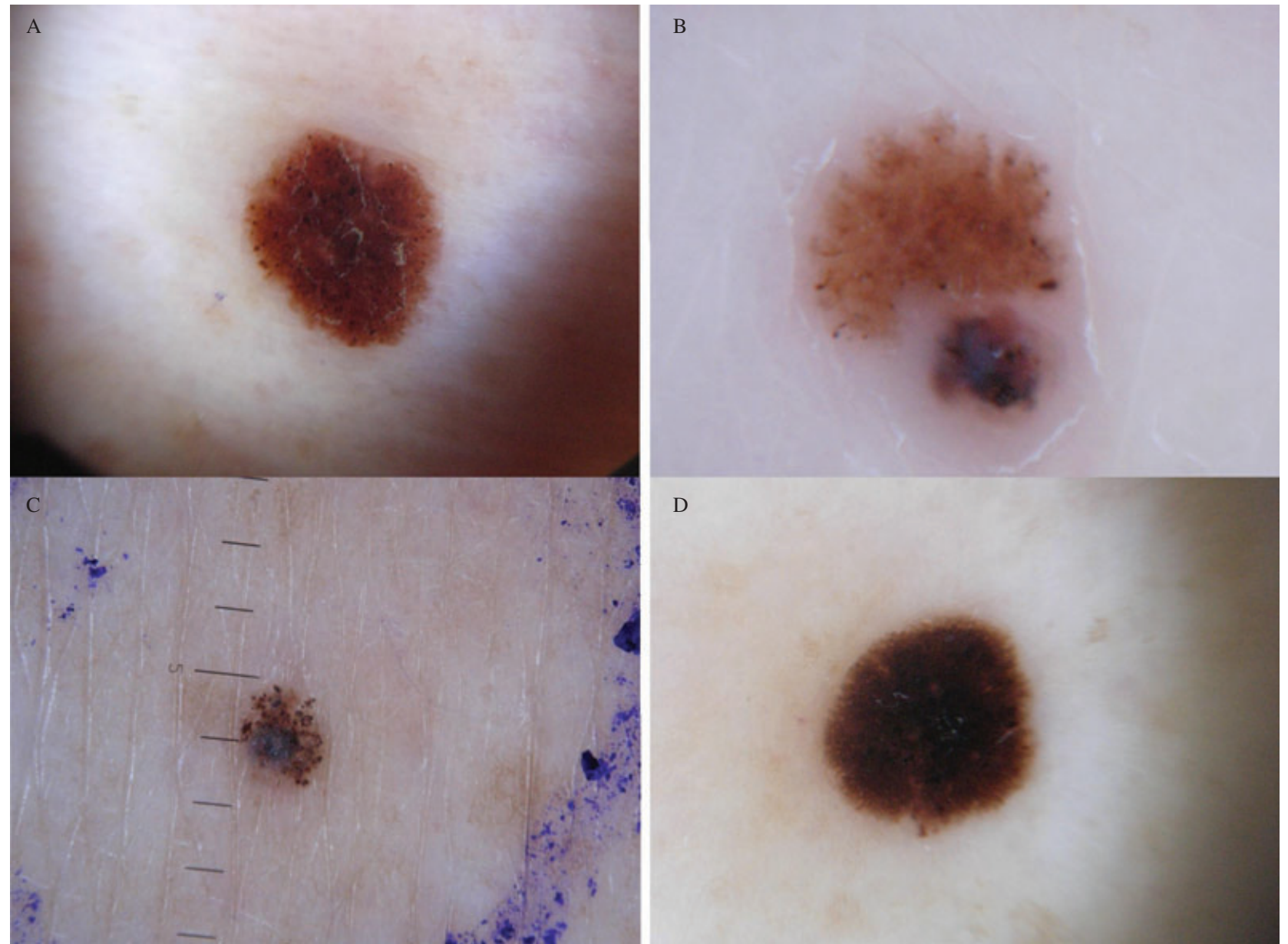

Fig. 6. Heterogeneous clinical and dermoscopic findings are depicted. There is a uniform diffuse brown lesion with a few darker dots throughout and at its periphery (A). A multicomponent pattern lesion has significant asymmetry of color and structure; blue-white structures, irregular dots and irregular blotches are seen (B). Depicted is a globular pattern lesion with variously sized dark brown globules; a blue-white veil is also noted at the periphery $(\mathrm{C})$. A regular and symmetric starburst pattern is also recognizable (D).

epidermotropic metastatic melanoma was suggested by clues expressed by most of our patient's lesions, including: (a) multiple disseminated lesions appearing in crops; (b) lesions of small size; and (c) lesional symmetry and circumscription.

Regression of the dermal component is unlikely to be the explanation of this epidermal-only pattern. Rather, the phenomenon of epidermotropic metastasis probably represents hematogenous spread with only transient or minimal dermal involvement. The survival of patients with melanoma is heavily dependent on the stage of the malignancy, and patients with epidermotropic metastases have a poor prognosis and distant metastases involving other organs are a frequent finding. ${ }^{14}$ PET combined with CT imaging (PET-CT) has become a major diagnostic tool in the detection of metastatic disease, as the technique improves the identification of deep soft tissue, lymph node and visceral metastases. ${ }^{15,16}$ PET-CT has been also proposed as the method of choice in detecting gastrointestinal melanoma metastases, which frequently are underdiagnosed by standard endoscopic techniques. ${ }^{17,18}$ However, small metastatic deposits need to achieve a certain size to be detected by PET-CT. ${ }^{15}$ In this case, the diagnosis of metastatic melanoma was particularly challenging. While the presence of widely disseminated small pigmented lesions appearing in crops in a patient with a previous diagnosis of melanoma suggested epidermotropic metastatic melanoma as a possibility, a staging evaluation (including a PET-CT) was negative for distant metastatic disease. In addition, histopathology was unable to discriminate epidermotropic metastases from multiple melanomas in situ and dermoscopy also failed to show unequivocal dermoscopic melanoma criteria. Indeed, few studies are available regarding the dermoscopic features of cutaneous metastatic melanoma. ${ }^{19-22}$ In a study with 130 cutaneous melanoma metastases, the saccular and vascular patterns as well as the presence of pigmentary halo and peripheral gray spots have been proposed as the most significant dermoscopic 


\section{Intraepidermal epidermotropic metastatic melanoma}
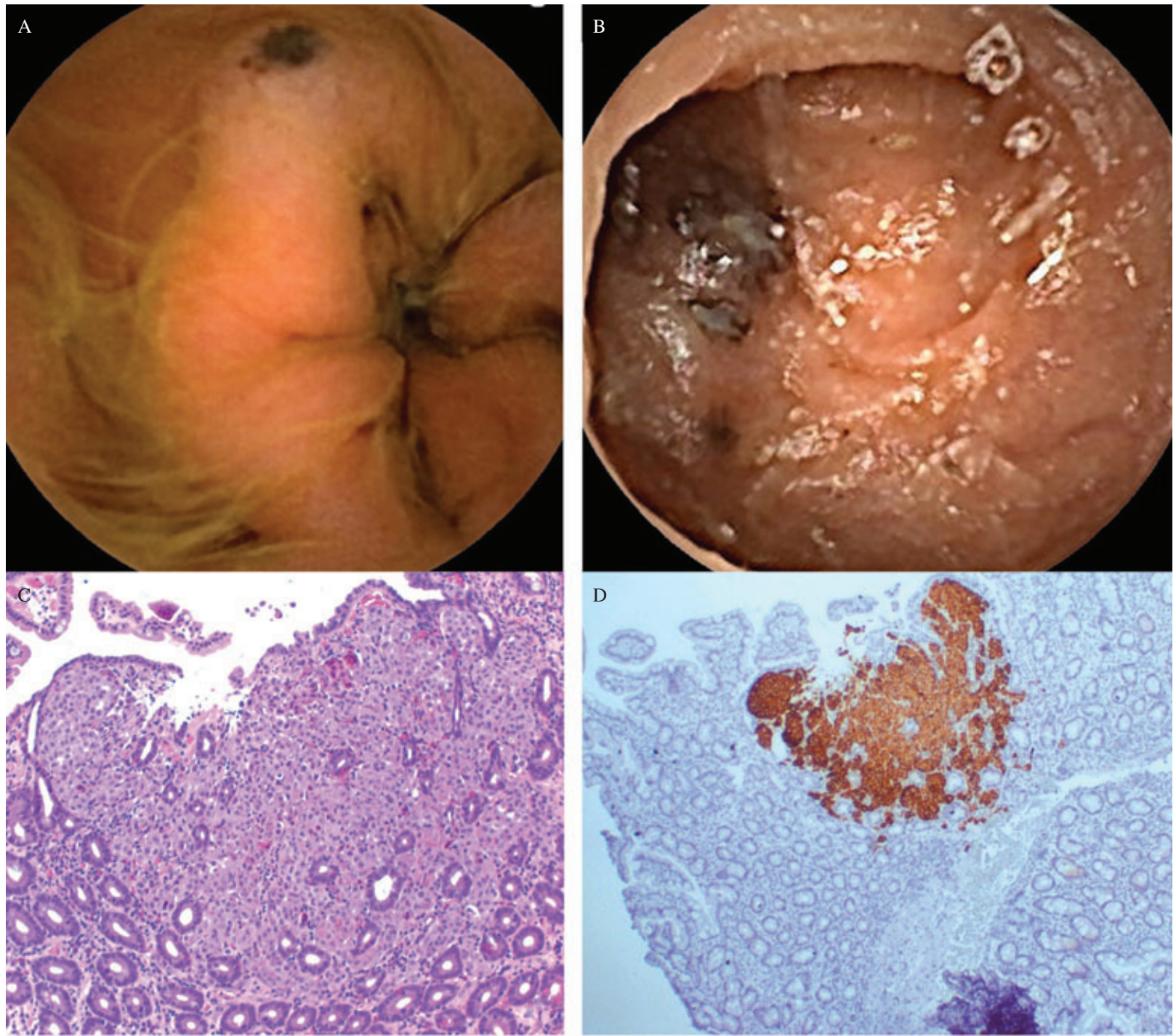

Fig. 7. Gastrointestinal melanoma metastases are depicted. The endoscopic appearance of gastrointestinal lesions shows dark-brown macules and ulcerated nodules. The histopathological features of a gastric melanoma metastasis include a proliferation of atypical melanocytes arranged in confluent nests in the superficial mucosa (hematoxylin and eosin, $\times 40$ ); positive immunostaining with HMB-45 was also observed.

elements supporting interpretation as metastatic melanoma. ${ }^{19}$ To the best of our knowledge, the dermoscopic results of epidermotropic metastatic melanoma have not been previously described. In our case, the dermoscopic findings were polymorphic and included reticular, homogeneous, globular and spitzoid patterns simulating melanocytic nevi or primary cutaneous melanoma. These heterogeneous findings suggest that the diagnosis of epidermotropic metastatic melanoma cannot reliably be made on the basis of dermoscopic features alone.

Genetic analysis of melanoma can provide additional information of use in the distinction of metastatic lesions from independent primaries. ${ }^{23-26}$ Although genetic intra- and intertumoral heterogeneity is a frequent feature in melanoma, it would be expected that a primary melanoma and its metastases would share one or more genetic alterations. Loss of heterozygosity (LOH) and X-chromosome inactivation analysis studies suggest that metastatic lesions in most melanoma patients are clonally related to their primary lesions. ${ }^{23,25,27}$ In our patient's tissue, the somatic mutation $\mathrm{V} 600 \mathrm{E}$ in the $B R A F$ gene and monosomy 9 were identified in the primary melanoma. Mutations in BRAF have been described in $40-80 \%$ of melanomas and V600E is the most common observed mutation. Mutations in BRAF appear to represent an early event in melanoma progression and are usually retained in secondary lesions. ${ }^{23,28}$ In our case, the V600E mutation was not initially detected in any of the seven freshly collected metastases, which were analyzed without macrodissection (and thus contaminating normal tissue may have obscured the molecular results). However, the subsequent use of FISH in paraffinembedded tissue sections and molecular analysis 


\section{Lestre et al.}

after macrodissection to avoid contamination allowed the demonstration of a molecular relationship between five presumed epidermotropic metastases and the original primary melanoma. No evidence of hereditary predisposition associated with germline mutations of $C D K N 2 A$ or $C D K 4$, as can be seen in familial and multiple primary melanoma, was found. ${ }^{29,30}$.

We therefore conclude that the 46 pigmented lesions affecting our patient represent intraepidermal epidermotropic metastatic melanoma and not multiple examples of primary melanoma in situ. The clinical appearance of the lesions in conjunction with atypical dermoscopic findings, the presence of multiple gastrointestinal metastases, and the genetic findings strengthen this assertion. Our case shows that histopathology alone can be insufficient to distinguish intraepidermal epidermotropic metastatic melanoma from multiple primary lesions. Its recognition should be based on correlation with clinical, dermoscopic, histopathological and genetic findings.

\section{References}

1. Miller AJ, Mihm MC. Melanoma. N Engl J Med 2006; 355: 51.

2. Zbytec B, Carlson JA, Granese J, Ross J, Mihm MC, Slominski A. Current concepts of metastasis in melanoma. Expert Rev Dermatol 2008; 3: 569.

3. Plaza JA, Torres-Cabala C, Evans H, Diwan HA, Suster S, Preito VG. Cutaneous metastases of malignant melanoma: a clinicopathologic study of 192 cases with emphasis in the morphologic spectrum. Am J Dermopathol 2010; 32: 129.

4. White WL, Hitchcock MG. Dying dogma: the pathological diagnosis of epidermotropic metastatic melanoma. Semin Diagn Pathol 1998; 15: 176.

5. Kornberg R, Harris M, Ackerman AB. Epidermotropically metastatic malignant melanoma: Differentiating malignant melanoma metastatic to the epidermis from malignant melanoma primary in the epidermis. Arch Dermatol 1978; 114: 67.

6. Bengoechea-Beeby MP, Velasco-Osés A, Fernández FM, Reguilón-Rivero C, RemónGarijo L, Casado-Pérez C. Epidermotropic metastatic melanoma: Are the current histologic criteria adequate to differentiate primary from metastatic melanoma? Cancer 2006; 72: 1909.

7. Gerami P, Shea C, Stone M. Angiotropism in epidermotropic metastatic melanoma: another clue to the diagnosis. Am J Dermatopathol 2006; 28: 429.

8. Piana S, Valli R, Ricci C. Epidermotropic metastasis of melanoma: report of a case of metastatic melanoma with previously unreported morphological features. Am J Dermatopathol 2009; 31: 301.

9. Allen AC, Spitz S. Malignant melanoma: a clinicopathological analysis of the criteria for diagnosis and prognosis. Cancer 1953; 6: 45.

10. Heenan PJ, Clay CD. Epidermotropic metastatic melanoma simulating multiple primary melanomas. Am J Dermotopathol 1991; 13: 396.
11. Moulin G, Franc M-P, Barrut D, Coiffet J, Viornery P. Métastases épidermotropes et jonctionnelles de mélanome malin. Ann Dermatol Venereol (Paris) 1980; 107: 1061.

12. Moreno A, Espanol I, Romagosa V. Angiotropic malignant melanoma. J Cutan Pathol 1992; 19: 325.

13. Abernethy JL, Soyer P, Kerl H, Jorizzo J, White WL. Epidermotropic metastatic malignant melanoma simulating melanoma in situ: a report of 10 examples from two patients. Am J Surg Pathol 1994; 18: 1140.

14. Savoia $\mathrm{P}$, Fava $\mathrm{P}$, Nardò $\mathrm{T}$, Osella-Abate S, Quaglino P, Bernengo MG. Skin metastases of malignant cutaneous melanoma: a clinical and prognostic survey. Melanoma Res 2009; 19: 321.

15. Kottschade LA, Suman VJ, Val Lowe, Morkovic SN. Positron emission tomography in early detection of relapse in high-risk melanoma. PET-TC relapse melanoma. Community Oncol 2009; 6: 344.

16. Strobel K, Dummer R, Husarik DB, Lago MO, Hany TF, Steinert HC. Highrisk melanoma: accuracy of FDG PET/CT with added CT morphologic information for detection of metastases. Radiology 2007; 244: 566.

17. Tatlidil R, Mandelkern M. FDG-PET in the detection of gastrointestinal metastases in melanoma. Melanoma Res 2001; 11: 297.

18. Reinhardt MJ, Joe AY, Jaeger U, et al. Diagnostic performance of whole body dual modality FDG PET/CT imaging for N- and Mstaging of malignant melanoma: experience with 250 consecutive patients. J Clin Oncol 2006; 24: 1178.

19. Bono R, Giampetruzzi AR, Concolino F, et al. Dermoscopic patterns of cutaneous melanoma metastases. Melanoma Res 2004; 14: 367.

20. Ferrari A, Peris K, Piccolo D, Chimenti S. Dermoscopic features of cutaneous local recurrent melanoma. J Am Acad Dermatol 2000; 43: 722.
21. Schultz H. Epiluminescence microscopy features of cutaneous malignant melanoma metastases. Melanoma Res 2000; 10: 273.

22. Pizzicheta MA, Canzonieri V, Gatti A, et al. Skin lesions in melanoma and Kaposi's sarcoma. Case 2: dermoscopic features of metastases from cutaneous melanoma mimicking benign nevi and primary melanoma. J Clin Oncol 2002; 20: 1412.

23. Rodolfo M, Daniotti M, Vallacchi V. Genetic progression of metastatic melanoma. Cancer Lett 2004; 214: 133.

24. Edinger JT, Radfar A, Judik DM. Two cutaneous malignant melanomas at the same anatomic site: a case report with molecular evaluation. J Cutan Pathol 2009; 36(Suppl. 1): 74 .

25. Orlow I, Tommasi D, Bloom B, et al. Evaluation of the clonal origin of multiple primary melanomas using molecular profiling. J Invest Dermatol 2009; 129: 1972.

26. Morita R, Fugimoto A, Hatta N, Takehara K, Takata M. Comparison of genetic profiles between primary melanomas and their metastases reveals genetic alterations and clonal evolution during progression. J Invest Dermatol 1998; 111: 919.

27. Bahrami S, Cheng L, Wang M, Jones TD, Malone JC, Billings SD. Clonal relationships between epidermotropic metastatic melanomas and their primary lesions: a loss of heterozygoty and X-chromosome inactivation-based analysis. Mod Pathol 2007; 20: 821 .

28. Rao UNM, Jones MW, Finkelstein SD. Genotypic analysis of primary and metastatic cutaneous melanoma. Cancer Genet Cytogenet 2003; 140: 37 .

29. Blackwood MA, Holmes R, Synnestvedt M, et al. Multiple primary melanoma revisited. Cancer 2002; 94: 2248.

30. Hayward NK. Genetics of melanoma predisposition. Oncogene 2003; 22: 3053. 
This document is a scanned copy of a printed document. No warranty is given about the accuracy of the copy. Users should refer to the original published version of the material. 